\title{
Effects of a Psychobiotic Supplement on Serum Brain-derived Neurotrophic Factor Levels in Depressive Patients: A Post Hoc Analysis of a Randomized Clinical Trial
}

\author{
Nazanin Heidarzadeh-Rad, ${ }^{1}$ Hülya Gökmen-Özel, ${ }^{1}$ Asma Kazemi, ${ }^{2 *}$ Negin Almasi, ${ }^{1}$ and Kurosh Djafarian ${ }^{3 *}$ \\ ${ }^{1}$ Department of Nutrition and Dietetics, Hacettepe University Faculty of Health Sciences, Ankara, Turkey; ${ }^{2}$ Nutrition Research Center, Shiraz \\ University of Medical Sciences, Shiraz, Iran; and ${ }^{3}$ Department of Clinical Nutrition, School of Nutritional Sciences and Dietetics, Tehran \\ University of Medical Sciences, Tehran, Iran
}

\begin{abstract}
Background/Aims
Psychobiotics are probiotics or prebiotics that, upon ingestion in adequate amounts, yield positive influence on mental health via microbiota-gut-brain axis regulation to modulate the circulating cytokines, chemokines, neurotransmitters, or neurotrophins levels. We have recently shown that a psychobiotic combination (Lactobacillus helveticus R0052 and Bifidobacterium longum R0175; CEREBIOME) significantly improved depression symptoms in patients with depression. Recent animal data suggest the influence of the gut microbiota on brain-derived neurotrophic factor (BDNF), which was shown to correlate with antidepressant response in depressive patients. Therefore, we conducted this exploratory post hoc analysis of BDNF levels to clarify the mechanism of action of this psychobiotic in our cohort.
\end{abstract}

\section{Methods}

Our study was a double-blind, randomized controlled trial of patients with low-to-moderate depression receiving either a probiotic combination, prebiotic or placebo. From the 110 patients randomized in the trial, 78 were included in this post hoc analysis (probiotic, $n=28$; prebiotic and placebo, $n=25$ ). We compared serum BDNF levels from participants at baseline and endpoint, and assessed the Pearson correlation between depression severity and BDNF levels for each intervention.

\section{Results}

We found that post-intervention BDNF levels were significantly different between groups $(P<0.001)$. Furthermore, BDNF levels increased significantly in the probiotic group compared to both the prebiotic $(P<0.001)$ and placebo groups $(P=0.021)$, which inversely correlated with depression severity compared to placebo (ANOVA/ANCOVA, $P=0.012 ;$ Pearson, $r=-0.79, P<0.001$ ). In the prebiotic group, BDNF levels reduced but not significantly compared with placebo group $(P>0.05)$.

\section{Conclusion}

Eight-week supplementation with B. longum and L. helveticus in depressive patients improved depression symptoms, possibly by increasing BDNF levels.

(J Neurogastroenterol Motil 2020;26:486-495)

Key Words

Brain-derived neurotrophic factor; Depression; Prebiotics; Probiotics 
Received: April 17, 2020 Revised: June 15, 2020 Accepted: July 21, 2020

(c) This is an Open Access article distributed under the terms of the Creative Commons Attribution Non-Commercial License (http://creativecommons. org/licenses/by-nc/4.0) which permits unrestricted non-commercial use, distribution, and reproduction in any medium, provided the original work is properly cited.

*Correspondence: Asma Kazemi and Kurosh Djafarian are equally responsible for this study.

Asma Kazemi, PhD

Nutrition Research Canter, Shiraz University of Medical Sciences, PO Box 71645-111, Shiraz, Iran

Tel: +98713612206, E-mail: kazemiasma66@gmail.com

Kurosh Djafarian, PhD

Department of Clinical Nutrition, School of Nutritional Sciences and Dietetic, Tehran University of Medical Sciences, No. 44, Hojjatdost Alley, Naderi St., Keshavarz Blvd, 14166/43931, Tehran, Iran

Tel: +98 912 6654577, Fax: +98 (0) 2188974462, E-mail: kdjafarian@tums.ac.ir

\section{Introduction}

Psychobiotics have been defined as probiotics, or support to probiotics (ie, prebiotics) that, upon ingestion in adequate amounts, yield positive influence on mental health. ${ }^{1,2}$ The effect of psychobiotics has been attributed to their impact on the microbiota-gut-brain axis (MGBA), which controls the bidirectional communication between the central nervous system (CNS) and the gut microbiota. ${ }^{2}$ A solid evidence base in animals now supports the notion that the modulatory effect of the MGBA on brain chemistry and function occurs via an alteration in parameters such as intestinal permeability and inflammation, or in the levels of neurotransmitters and other neurotrophic factors, such as serotonin or brain-derived neurotrophic factor $(\mathrm{BDNF})^{2}$

$\mathrm{BDNF}$, as a neurotrophin, regulates neurogenesis by promoting cell growth, proliferation, migration, differentiation, and death. BDNF by interacting with its specific receptor, the tyrosine kinase receptor B on CNS target cells interferes in important neurophysiological processes in both central and peripheral nervous systems, and supports neuronal survival ${ }^{3}$ as well as neuroplasticity and neuroprotection. ${ }^{4,5} \mathrm{BDNF}$ mediates the activation of various signaling pathways such as mitogen-activated protein kinase or phosphatidylinositol-3-kinase pathways, and leads to up-regulation of various neuroprotective proteins ${ }^{6}$ such as anti-apoptotic protein kinase B and B-cell lymphoma 2.,7

In recent years, several publications have documented the effects of the intestinal microbiota or probiotics on mood and anxiety in healthy individuals or in populations with depressive symptoms secondary to another condition. ${ }^{8-15}$ However, in psychiatric populations, studies about psychobiotics are limited.

Overall, as for all probiotics, the benefits and mechanisms of action of psychobiotics appear to be strain- and disease-specific. ${ }^{16,17}$ Furthermore, whether or not the mechanisms identified in animal models will translate to human psychiatric populations remains to be demonstrated. ${ }^{18}$ The current psychobiotic formulation (Lactobacillus helveticus R0052 and Bifidobacterium longum R0175) was shown to reduce anxiety and depression in healthy subjects compared with controls. ${ }^{13,19}$ Behavioral symptoms of stress and anxiety were improved by the psychobiotic formulation in rats, ${ }^{13}$ and behavioral symptoms of depression were improved in a post-myocardial infarction depression mouse model. ${ }^{20,21}$ This formulation was also shown to attenuate the effects of chronic stress in mice by preventing the increase in corticosterone, adrenaline, and noradrenaline, and the decrease in neurogenesis induced by the water avoidance test, notably by increasing the levels of BDNF in the hypothalamus. ${ }^{22}$

In humans, the mechanisms of action of psychobiotics on depression in major depressive disorder (MDD) patients remain largely uncharacterized. Hence, the aim of the current post hoc analysis is to assess the changes in serum BDNF levels in a cohort of MDD patients who received probiotic, prebiotic, or placebo for 8 weeks, and to gather mechanistic insights into the antidepressant effect of this psychobiotic in patients with low-to-moderate depression. ${ }^{23}$

\section{Materials and Methods}

\section{Study Design and Participants}

This exploratory post hoc analysis was conducted on the perprotocol (PP) population of a double blind, randomized, placebocontrolled trial (RCT) conducted between July 2016 and April $2018,^{23}$ which included 3 parallel arms: probiotics, prebiotics, and placebo. Eligible participants were randomized in a 1:1:1 ratio into 1 of the 3 arms and received the intervention for a period of 8 weeks. Ethics approval was provided by the research ethics committee of Tehran University of Medical Sciences (Registration No. IR.TUMS.VCR.REC.1396.4243) and the study was registered in IRCT.ir under IRCT2015092924271N1. The approved informed 
consent form was signed by all participants before their inclusion in the study. Notably, the informed consent form included the information that the samples will be stored for further use in follow-up analyses.

The study population was described previously. ${ }^{23}$ Briefly, out of the 230 patients screened following referral by a psychiatrist of the psychiatric clinic of the Bahman Hospital (Tehran, Iran), 110 patients (78 women and 32 men) with MDD were included in the trial. Volunteers included in the RCT were aged between 20 years and 50 years, had a current diagnosis of mild to moderate melancholic depression for at least 1 month, and were taking one of the following anti-depressant medications: fluoxetine, citalopram, amitriptyline, or sertraline. Exclusion criteria included any sensitivity reaction to prebiotic and probiotic compounds, refusal to cooperate, any serious changes in diet routine and lifestyle during the study, any changes in medication or its dosage, long term (at least 1 week) inflammatory disease requiring anti-inflammatory pharmacotherapy, pregnancy or lactation, antibiotic intake during the study, history of cancer, diabetes, pancreatitis, or thyroid, kidney, liver, respiratory, or cardiovascular disorders, diagnosis of nutritional allergy by a medical professional, regular consumption of probiotic products within 2 months of study start, dietary supplement intake such as vitamins, antioxidant and/or omega-3's at least 4-6 weeks before the study, alcohol consumption (alcoholism according to Diagnostic and Statistical Manual of Mental Disorders-IV criteria), smoking (at least 5 cigarettes per day during last 6 months or pipe or hookah at least once in last month), opiate addiction or substance abuse, history of heart attack or stroke, following a specific diet (such as vegetarianism, etc), using hormonal drugs and who participated in another study in the 2 months preceding the study. Participants were instructed to avoid consumption of any other probiotic supplements or vitamins during the study.

As previously described, the PP population of the main trial was composed of 81 participants (probiotics, $n=28$; prebiotic, $n$ $=27$; and placebo, $n=26$ ). However, for 3 participants, the volume of serum obtained at the last blood collection was insufficient. Hence, for consistency, all analyses, including the Beck's Depression Inventory (BDI) score, were performed here on the individuals from the PP population for whom BDNF levels were available at both time points (all, $\mathrm{n}=78$; probiotics, $\mathrm{n}=28$; prebiotic, $\mathrm{n}=$ 25 ; and placebo, $\mathrm{n}=25)$ (Supplementary Figure).

\section{Randomization and Blinding}

As previously described, ${ }^{23}$ randomization was performed by a research assistant not taking part in the project using the method available in www.randomization.com. A permuted block randomization was stratified by ( $\geq 35$ vs $<35$ ). Intervention packs (probiotic, prebiotic, and placebo sachets) were pre-packed and assigned to a code according to the randomization sequence. The codes were concealed from all participants and researchers until the end of the data analysis.

\section{Interventional Products}

Interventional products were formulated and provided by Lallemand Health Solutions Inc (Mirabel, Quebec, Canada). All supplements were manufactured as orally dispersible powders with similar appearance and taste, and were packaged in $5 \mathrm{~g}$ sachets containing 10 billion ( $\left.\geq 10 \times 10^{9}\right)$ CFU of freeze-dried L. helveticus R0052 (CNCM strain I-1722) and B. longum R0175 (CNCM strain I-3470) along with excipients (xylitol, maltodextrin, malic acid, and plum flavor). The prebiotic sachets contained $80 \%$ galactalogosaccharide (GOS) powder per sachet along with the excipients while the placebo sachets contained only the excipients.

Participants were instructed to consume 1 sachet at the same time daily for 8 weeks by directly pouring the powder on the tongue. The remaining sachets were counted at the end of the trial to assess compliance. Subjects were deemed compliant if they consumed a minimum of $80 \%$ of the sachets.

\section{Outcome Measures}

Except for BDNF levels measurements, outcome measures were described previously. ${ }^{23}$ Demographic and general characteristics of the participants, body weight and height measurements, nutritional status, depression severity, and physical activity levels were acquired at the beginning and end of the trial. The nutritional status was evaluated based on 3 dietary records ( 2 weekdays and 1 weekend) using the software Nutritionist 4 (First Databank, San Bruno, CA, USA) adapted for Iranian foods.

The depression status was measured using the BDI. The BDI is a validated self-reporting scale measuring the emotional, cognitive, somatic, and motivational components of depression. The latest version of the inventory, BDI-II, has been validated among the Iranian population. ${ }^{24}$ This scale is composed of 21 questions related to a specific symptom of depression, each consisting of 4 answers that express the severity of the symptom, thereby generating a score between 0 and 3 for each question. The sum of all scores indicates BDI score of each participant. The higher total score relates to the more severe grade of depression.

Physical activity was evaluated using the Short International Physical Activity Questionnaire and defined as metabolic equiva- 
lents. It was calculated by multiplying the duration of each activity with its specific coefficient. Height and fasting body weight with minimal clothing and no shoes were acquired using a digital scale. The body mass index (BMI, $\mathrm{kg} / \mathrm{m}^{2}$ ) was calculated by dividing the weight $(\mathrm{kg})$ by the squared height $\left(\mathrm{m}^{2}\right)$.

Blood samples $(10 \mathrm{~mL})$ were collected in the fasting state, between $8 \mathrm{AM}$ and $9 \mathrm{AM}$, at the beginning and end of the 8-week trial. Briefly, blood samples were transferred to test tubes and were immediately centrifuged at $3500 \mathrm{rpm}$ for 10 minutes to extract the serum. Aliquoted serum samples were readily frozen and kept at $-80^{\circ} \mathrm{C}$ until further analysis. Serum BDNF levels were measured using a commercially available enzyme-linked immunosorbent assay kit (Shanghai Crystal Day Biotech Co, Ltd, Shanghai, China) according to the manufacturer's instructions.

\section{Statistical Methods}

Statistical analyses of the data obtained in this study were performed using the Statistical Package for Social Science (IBM SPSS, version 22.0; IBM, Armonk, NY, USA). For this post hoc analysis, all outcome measures were analyzed according to the PP principle for the participants who had BDNF levels measured at both time points $(\mathrm{n}=78)$. Data normality was analyzed by KolmogorovSmirnov test. ANOVA/ANCOVA were used for comparing the values between groups adjusting for corresponding baseline values. ANOVA/ANCOVA were also used for pairwise comparisons between groups. Quantitative variables were described as the mean \pm standard deviation (SD), and categorical variables were expressed as frequency (percentage). Normally distributed variables were reported as the mean $\pm \mathrm{SD}$. For non-normally distributed data, the STATA software version 13.0 (StataCorp LP, College Station, TX, USA) was used to apply the appropriate transformation method; a square root transformation was used for BDI scores and a logarithmic transformation was used for the changes in BDNF levels and selenium intake. Data were retransformed to the mean $\pm 95 \%$ confidence intervals. ANOVA/ANCOVA test was used to confirm significance between adjusted means. All tests for significance were two-sided and values of $P<0.05$ were considered statistically significant.

As described previously, ${ }^{23}$ the trial sample size was determined based on the mean reductions in BDI score obtained in a similar study conducted in a population of patients. ${ }^{25}$ It was calculated as 27 subjects per group, considering a power of $80 \%$, alpha error of 0.05 and $10 \%$ possible lost to follow-up to have mean difference of 5 in BDI scores between intervention and placebo groups. To compensate for unexpected loss to follow-up rates, 110 subjects were included in the initial trial.

\section{Results}

As previously described, out of the 110 participants included in the trial and randomized, a total of 32 participants did not complete the study: 10 in the probiotics group, 9 in the prebiotic group, and

Table 1. Baseline Characteristics of the Post Hoc Population

\begin{tabular}{|c|c|c|c|c|c|}
\hline Characteristics & Probiotic $(\mathrm{n}=28)$ & Prebiotic $(n=25)$ & Placebo $(\mathrm{n}=25)$ & Total $(\mathrm{N}=78)$ & $P$-value \\
\hline Age (yr) & $37.8 \pm 7.9$ & $36.6 \pm 8.4$ & $36.0 \pm 8.5$ & $36.0 \pm 9.0$ & 0.460 \\
\hline Sex & & & & & 0.490 \\
\hline Woman & $20.0(71.4)$ & $20.0(80.0)$ & $15.0(60.0)$ & $55.0(70.5)$ & \\
\hline Man & $8.0(28.6)$ & $5.0(20.0)$ & $10.0(40.0)$ & $23.0(29.5)$ & \\
\hline Weight (kg) & $72.2 \pm 11.8$ & $73.0 \pm 15.8$ & $73.4 \pm 13.0$ & $72.8 \pm 13.4$ & 0.950 \\
\hline $\operatorname{BMI}\left(\mathrm{kg} / \mathrm{m}^{2}\right)$ & $26.6 \pm 4.2$ & $27.2 \pm 5.1$ & $26.8 \pm 4.5$ & $26.9 \pm 4.5$ & 0.850 \\
\hline Duration of depression $(\mathrm{yr})^{\mathrm{a}}$ & $1.3 \pm 1.8$ & $1.5 \pm 1.7$ & $1.4 \pm 1.8$ & $1.8 \pm 1.8$ & 0.661 \\
\hline $\begin{array}{l}\text { Duration of antidepressant } \\
\text { therapy }(\mathrm{yr})^{\mathrm{a}}\end{array}$ & $1.3 \pm 4.5$ & $2.1 \pm 3.9$ & $1.5 \pm 4.1$ & $1.6 \pm 4.2$ & 0.960 \\
\hline Antidepressant medication & & & & & 0.211 \\
\hline Citalopram & $5.0 \pm 17.8$ & $1.0 \pm 4.0$ & $8.0 \pm 30.0$ & $14.0 \pm 17.0$ & \\
\hline Fluoxetine & $9.0 \pm 32.0$ & $4.0 \pm 16.0$ & $2.0 \pm 8.0$ & $15.0 \pm 19.2$ & \\
\hline Sertraline & $8.0 \pm 28.6$ & $14.0 \pm 52.0$ & $8.0 \pm 30.0$ & $30.0 \pm 38.5$ & \\
\hline Amitriptyline & $4.0 \pm 14.0$ & $5.0 \pm 20.0$ & $6.0 \pm 23.0$ & $15.0 \pm 19.2$ & \\
\hline BDI scores ${ }^{b}$ & $17.6(14.2-21.5)$ & $19.7(15.9-24.0)$ & $18.2(14.3-22.5)$ & $18.5(3.9-22.8)$ & 0.730 \\
\hline
\end{tabular}

BMI, body mass index; BDI, Beck's Depression Inventory.

Non-normal data were logarithmic ${ }^{a}$ or square root ${ }^{b}$ transformed and retransformation was performed to display as mean (95\% CI).

The quantitative variables are expressed as mean $\pm \mathrm{SD}$, number $(\%)$, or mean $(95 \% \mathrm{CI})$. 
Table 2. Serum Brain-derived Neurotrophic Factor Levels of the Participants at Baseline and Endpoint

\begin{tabular}{|c|c|c|c|c|}
\hline Time of measure & Probiotic $(\mathrm{n}=28)$ & Prebiotic $(\mathrm{n}=25)$ & Placebo $(n=25)$ & $P$-value \\
\hline Baseline (ng/mL) & $0.392(0.346-0.445)$ & $0.425(0.367-0.492)$ & $0.483(0.419-0.558)$ & 0.090 \\
\hline Endpoint (ng/mL) & $0.483(0.424-0.548)$ & $0.389(0.335-0.453)$ & $0.467(0.401-0.542)$ & $\begin{array}{r}0.090^{\mathrm{a}} \\
<0.001^{\mathrm{b}}\end{array}$ \\
\hline$\Delta \mathrm{BDNF}(\mathrm{ng} / \mathrm{mL})$ & $\begin{array}{c}0.095 \\
(0.056-0.134)\end{array}$ & $\begin{array}{c}-0.056 \\
(-0.096--0.010)\end{array}$ & $\begin{array}{c}0.009 \\
(-0.047--0.039)\end{array}$ & $\begin{array}{c}<0.001 \\
\text { Partial eta } \\
\text { square } \\
0.262\end{array}$ \\
\hline
\end{tabular}

${ }^{a}$ Not adjusted and ${ }^{b}$ adjusted for baseline values.

$\triangle \mathrm{BDNF}$, change from baseline.

Data for serum brain-derived neurotrophic factor (BDNF) levels were log transformed and then back transformed to display as mean (95\% CI).

$P$-value is based on ANOVA/ANCOVA test. $P<0.05$ is considered significant. Partial eta squared is an effect size measure for one-way or factorial ANOVA.

10 in the placebo group discontinued the study or were lost to follow up. In addition, for 2 participants in prebiotic and 1 in placebo groups, an insufficient volume serum was recovered at the final time point (8 weeks). Overall, compliance was high, with an average (SD) of $91.90 \%$ (5.53\%) of the supplements consumed by the participants throughout the study.

\section{Baseline Characteristics}

The baseline characteristics of the 78 participants included in the post hoc analyses are summarized in Table 1 . The majority of participants, $70.5 \%$, were women. The mean $\pm \mathrm{SD}$ age of the participants was $36.0 \pm 9.0$ years. There was no significant difference between the 3 groups in terms of general characteristics at baseline $(P$ $>0.05)$. The mean BMI of the participants was $26.9 \pm 4.5$ and there was no significant difference between the groups. The participants were MDD patients for an average of $1.8 \pm 1.8$ years and were receiving antidepressant medications for an average of $1.6 \pm$ 4.2 years. The participants in each group were not different in terms of weight, BMI, duration of depression, duration of antidepressant therapy, and BDI scores at baseline $(P>0.05)$.

\section{Serum Brain-derived Neurotrophic Factor Levels}

We compared the changes in BDNF levels from baseline to the endpoint for the post hoc PP population (Table 2). The changes in serum BDNF levels were significantly different between the study groups using ANOVA/ANCOVA $(P<0.001)$, with an increase in the probiotic group $(0.095[0.056-0.134])$ and placebo group $(0.009[-0.047-0.039])$, and a decrease in the prebiotic group $(-0.056[-0.096-0.010])$.

Pairwise comparisons of the groups, shown in Table 3, revealed that the increase in BDNF levels in the probiotic group was statistically significant compared to both the prebiotic $(P<0.001)$ and
Table 3. Pairwise Comparison of the Serum Brain-derived Neurotrophic Factor Levels Over the Supplementation Period for All Groups

\begin{tabular}{lccc}
\hline $\begin{array}{c}\text { Statistical } \\
\text { measures }\end{array}$ & $\begin{array}{c}\text { Probiotic and } \\
\text { Prebiotic }\end{array}$ & $\begin{array}{c}\text { Probiotic and } \\
\text { Placebo }\end{array}$ & $\begin{array}{c}\text { Prebiotic and } \\
\text { Placebo }\end{array}$ \\
\hline $\begin{array}{l}P \text {-value } \\
\text { Cohen's d }\end{array}$ & $<0.001$ & 0.021 & 0.080 \\
$P$
\end{tabular}

$P<0.05$ is considered significant. Based on ANOVA/ANCOVA test adjusted for baseline values.

placebo $(P=0.021)$ groups, while a slight decrease observed in the prebiotic group when compared to placebo $(P=0.080)$.

\section{Anthropometric Measurements, Dietary Intake, and Physical Activity}

Based on ANOVA/ANCOVA test, there was no difference in weight and BMI among groups at the endpoint $(P=0.940$ and $P$ $=0.910$, respectively) (Table 4 ). Dietary intake and physical activity were not significantly different between groups at the end of the study relative to baseline values (Supplementary Table 1).

Despite the lack of significance in some parameters that were previously found different in the intention-to-treat (ITT) population, when compared by pairwise analyses, selenium intake was significantly lower in the probiotic group in comparison to the prebiotic group $(P=0.030)$. Additionally, energy intake in the prebiotic group was significantly lower than both probiotic and placebo groups ( $P=0.040$ and $P=0.020$, respectively) (Supplementary Table 2).

\section{Beck's Depression Inventory Scores}

BDI scores analysis was based on the post hoc PP population ( $\mathrm{n}$ $=78$ ) (Table 4). ANOVA/ANCOVA test showed that, similarly 
Table 4. Anthropometric Measurements and Beck's Depression Inventory Scores of the Post Hoc Population at Baseline and Endpoint

\begin{tabular}{|c|c|c|c|c|c|c|c|}
\hline \multirow{2}{*}{ Variables } & \multicolumn{2}{|c|}{ Probiotic $(n=28)$} & \multicolumn{2}{|c|}{ Prebiotic $(n=25)$} & \multicolumn{2}{|c|}{ Placebo $(n=25)$} & \multirow{2}{*}{$P$-value } \\
\hline & Baseline & End & Baseline & End & Baseline & End & \\
\hline Weight $(\mathrm{kg})$ & $72.2 \pm 11.8$ & $72.8 \pm 12.7$ & $73.0 \pm 15.8$ & $71.9 \pm 16.2$ & $73.4 \pm 13.0$ & $73.5 \pm 13.9$ & 0.940 \\
\hline $\operatorname{BMI}\left(\mathrm{kg} / \mathrm{m}^{2}\right)$ & $26.6 \pm 4.2$ & $26.5 \pm 4.3$ & $27.2 \pm 5.1$ & $26.8 \pm 5.1$ & $26.8 \pm 4.5$ & $27.1 \pm 4.6$ & 0.910 \\
\hline BDI scores & $17.6 \pm 9.9$ & $9.8 \pm 8.0$ & $19.7 \pm 10.3$ & $14.1 \pm 13.5$ & $18.2 \pm 10.4$ & $15.9 \pm 12.7$ & 0.044 \\
\hline$\Delta \mathrm{BDI}$ score & \multicolumn{2}{|c|}{$-7.5 \pm 7.8$} & \multicolumn{2}{|c|}{$-4.7 \pm 8.6$} & \multicolumn{2}{|c|}{$-2.1 \pm 6.7$} & 0.036 \\
\hline
\end{tabular}

BMI, body mass index; BDI, Beck's Depression Inventory; $\triangle \mathrm{BDI}$, BDI change from baseline.

BDI score was square root transformed. Data are expressed as mean $\pm \mathrm{SD}$.

$P$-values obtained from ANOVA/ANCOVA adjusted for baseline values. $P<0.05$ is considered significant.

to what was seen in the ITT population, ${ }^{23}$ the BDI scores assessed at the beginning and end of the trial were significantly different between the study groups $(P=0.044)$.

Furthermore, in accordance with the previously published ITT population analyses, ${ }^{23}$ pairwise comparisons for the post hoc population revealed that a significant decrease in the BDI score was observed only in the probiotic group compared to placebo group $(P=0.012)$ (Table 5). There was no significant difference between probiotic and prebiotic, nor prebiotic and placebo supplementations $(P>0.05)$.

Furthermore, the BDNF levels and change in BDI scores over the supplementation period were inversely correlated in our population $(\mathrm{n}=78)$. When comparing the correlation between these parameters among the 3 groups, we observed a stronger correlation between BDNF levels and BDI score changes in the probiotics group $(r=-0.79, P<0.001)$ compared to prebiotic $(r=-0.19$, $P=0.190)$ or placebo group $(r=-0.69, P=0.001)$.

\section{Adverse Events}

Some adverse events were reported by participants that may be related to the intervention. The most common adverse event was increased appetite, which was reported by 5 participants in the probiotic group and 1 participant in the prebiotic group. This reported adverse event may be reflecting beneficial effect, which was further investigated in a post- hoc analysis. ${ }^{26}$ Gastrointestinal complaints were the most reported adverse event in the prebiotic group (4 reports); it was also reported by two participants in the probiotic group. Nausea was reported by 1 in the probiotic group and 1 in the prebiotic group. Also 1 participant in the probiotic group reported fever and body aches. There was no adverse event in the placebo group except for 1 report of worse mental state, which was also reported by 1 participant in the probiotic group but was deemed unrelated to the intervention.
Table 5. Pairwise Comparison of the Beck's Depression Inventory Score Changes of the Post Hoc Population Over the Supplementation Period

\begin{tabular}{lccc}
\hline $\begin{array}{c}\text { Statistical } \\
\text { measures }\end{array}$ & $\begin{array}{c}\text { Probiotic and } \\
\text { Prebiotic }\end{array}$ & $\begin{array}{c}\text { Probiotic and } \\
\text { Placebo }\end{array}$ & $\begin{array}{c}\text { Prebiotic and } \\
\text { Placebo }\end{array}$ \\
\hline $\begin{array}{l}\text { P-Value } \\
\text { Cohen's d }\end{array}$ & 0.180 & 0.012 & 0.260 \\
\hline
\end{tabular}

$P<0.05$ is considered significant. Cohen's d effect size based on ANOVA/ ANCOVA test adjusted for baseline.

\section{Discussion}

The main goal of this post hoc analysis of a double-blind, randomized placebo-controlled trial was to gather additional insight into the potential mechanisms of action behind the beneficial effects of the psychobiotic formulation containing $L$. helveticus R0052 and B. longum R0175 on depressive symptoms in a psychiatric population. To this end, we assessed the changes in serum BDNF levels following probiotic and prebiotic supplementation in $78 \mathrm{MDD}$ patients who were randomly divided into 3 groups, probiotic ( $\mathrm{n}$ $=28)$, prebiotic $(n=25)$, and placebo $(n=25)$. The findings of the current post hoc analysis are: (1) post-intervention BDNF levels in the serum were significantly higher in the probiotics group compared with prebiotic and placebo groups, and (2) The changes in serum BDNF levels and BDI score over the 8 weeks of supplementation were inversely correlated, with a significantly stronger correlation in the probiotics group. Indeed, and as was seen in the ITT population, ${ }^{23}$ BDI scores were significantly reduced in the probiotic-supplemented group compared to the placebo group when considering the post hoc $\mathrm{PP}$ population, while the reduction in the BDI scores of the prebiotic group was not different from placebo. In the initial analyses on the ITT population, the mean BDI score was also only reduced significantly in the probiotic group compared 
to placebo $\left(P\right.$-value $_{(\mathrm{ITT})}=0.008 ; P$-value $\left._{(\mathrm{PP})}=0.012\right)$, but not in the prebiotic group. Interestingly, BDNF levels were shown to be correlated with the response of patients to antidepressants rather than to the severity of depression, which suggests that BDNF may be essential for antidepressant response. ${ }^{27,28}$ The fact that Romijn et $\mathrm{al}^{29}$ observed no effect of this psychobiotic on BDNF levels in medication-free depressed patients argues for a role of this formulation in enhancing the response to antidepressants, possibly by increasing BDNF levels. Importantly, this formulation also increased BDNF levels in mice models of stress induced by water avoidance, ${ }^{22}$ so this is an example where a mechanism identified in an animal model appears to translate to humans.

Our findings are consistent with the studies evaluating the effects of these probiotics on BDNF levels in animal models. ${ }^{12,22,30}$ Liang et $\mathrm{al}^{30}$ found that $L$. helveticus NS8 administration in rats with chronic stress resulted in more hippocampal BDNF mRNA, as did citalopram, suggesting possible antidepressant-like effects of $L$. helveticus, especially in stress related depression. ${ }^{30}$ In a study evaluating the effect of $L$. helveticus R0052 and B. longum R0175 on hypothalamic pituitary adrenal response in mice exposed to stress, it was shown that pretreatment with the probiotic formulation reversed the stress-induced reduction of BDNF expression and also corticosterone, noradrenaline, and adrenaline increase in mice. ${ }^{22} \mathrm{~B}$. longum administration to rats with anxiety-like behavior improved their behavior and restored hippocampal BDNF mRNA levels, without influencing the cytokine profile, highlighting a BDNFdependent and cytokine-independent mechanism mediating the anxiolytic effects of $B$. longum. ${ }^{12}$ However, another study has supported the anxiolytic properties of this bacterium in murine models, but without any significant effect on neural BDNF expression. ${ }^{31}$ The authors have suggested that probiotic fermentation products interact with enteric neurons and regulate the enteric nervous system excitability, so it presents its effects on the CNS through vagal pathways. Although assessing brain BDNF in humans is not possible, but as BDNF passes through blood-brain barrier, peripheral blood BDNF levels mirror brain-tissue BDNF levels and is positively correlated with BDNF in the CNS. ${ }^{32,33}$

As studies on animal models appear to point towards a role of BDNF in the effects of probiotics, studies exploring this hypothesis in depressive patients are limited. ${ }^{34}$ In a pilot study on irritable bowel syndrome patients, $B$. longum supplementation for 6 weeks lowered depression scores but without affecting serum BDNF levels in either the probiotic or placebo groups, although the possibility of the probiotic regulating BDNF expression in some parts of the brain was not excluded..$^{34}$ Moreover a recent RCT in hemodialysis patients compared the effects of a 3 month supplementation with either a control, a probiotics (blend of Lactobacillus acidophilus, B. longum, Bifidobacterium bifidum, and Bifidobacterium lactis) or a synbiotic formulation (including the same 4 probiotic bacteria with 3 prebiotics (fructooligosaccharide, GOS, and inulin). ${ }^{35}$ While depression scores improved by both supplements with more effectiveness of synbiotics, serum BDNF levels increased significantly with synbiotic supplementation compared to probiotics, particularly in the depressed patients subgroup. The authors linked the greater effectiveness of synbiotic in patients with end-stage renal disease to uremia-induced dysfunction of intestinal epithelial barrier which may inhibit the probiotics alone to show their microbial modulatory effects. ${ }^{36}$ However, in contrast to our findings, Chung et al ${ }^{37}$ found no significant effect of $L$. helveticus IDCC3801 fermented milk administration on geriatric depression scale, perceived stress scale, and BDNF levels of healthy older individuals. Our findings on BDI scores seems to be consistent with other study in MDD patients which found that 8-week supplementation of probiotic capsules (consisting L. acidophilus, Lactobacillus casei, and B. bifidum) resulted in greater reduction in BDI scores compared to placebo. ${ }^{38}$ In line with our findings, Messaoudi et $\mathrm{al}^{13}$ found that administration of probiotic formulation (L. helveticus R0052 and B. longum R0175) improved depression scores in healthy volunteers and anxiety-like behavior in rats. Similarly, in another study on healthy individuals the same probiotic formulation was beneficial on psychological distress, even with low urinary free cortisol levels. It was concluded that prophylactic use of probiotics can improve mood regardless of the stress level. ${ }^{39}$

Here we show that prebiotic GOS administration did not modify BDNF levels significantly in this population, in accordance with the absence of a significant effect on the BDI score. Despite this, it is likely that GOS may have affected the composition of the gut microbiota. A dose of $5 \mathrm{~g}$ of GOS was shown to affect microbiota composition in healthy adults, essentially by promoting Bifidobacteria. ${ }^{40}$ In this study microbiota composition of the participants was not analyzed. The changes in microbiota composition associated with MDD are not yet fully characterized, but differences have been identified compared to healthy individuals. ${ }^{41}$ Notably, Bifidobacteria, Faecalibacterium, and Dialister were shown to be decreased while Bacteroides and Blautia are frequently increased in MDD patients. Importantly, microbiota composition was shown to be affected by antidepressants, which have been shown to possess an antibacterial effect postulated as being part of their mode of action. ${ }^{42}$ While the overall effect of GOS on clinical parameters was not significant, an effect of this prebiotic on microbiota composition that 

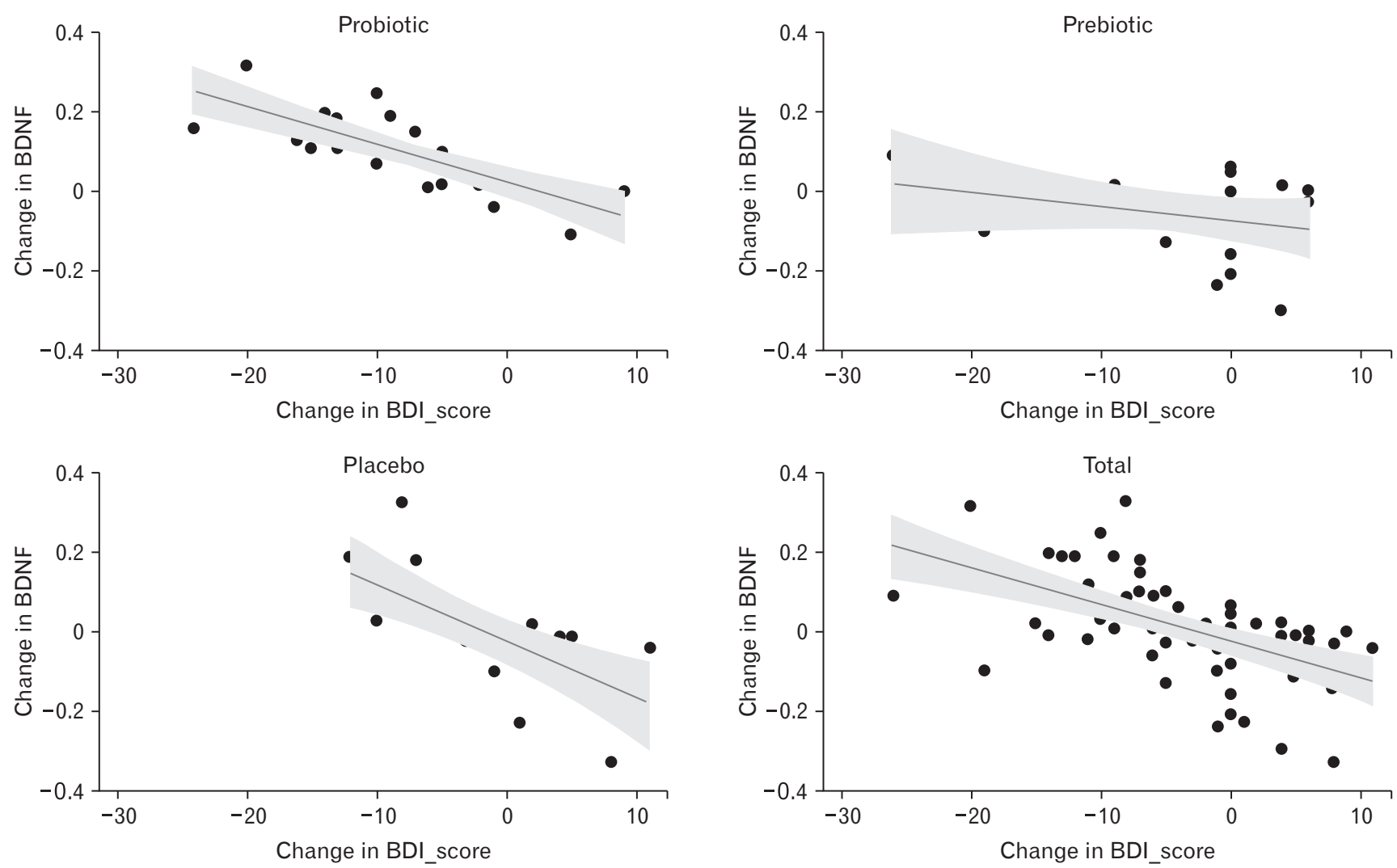

Figure. Pearson correlation analyses between brain-derived neurotrophic factor (BDNF) levels and Beck's Depression Inventory (BDI) score changes among the 3 groups. X-axis represents BDI score changes and y-axis represents BDNF changes. As shown, there is negative correlation between the change in BDNF levels and BDI scores over 8-week supplementation period, while the correlation in probiotic group $(r=-0.79, P$ $<0.001)$ is larger compared to prebiotic group $(r=-0.19, P=0.190)$ or placebo group $(r=-0.69, P=0.001)$.

could modulate selective serotonin reuptake inhibitor (SSRI) activity or metabolism cannot be excluded. ${ }^{41}$ The apparent difference in the distribution of participants in the prebiotic group with regards to the correlation between BDI change and BDNF levels (Figure), even if not significantly different from placebo or probiotic, may justify further studies on the effects of GOS on microbiota composition in MDD patients taking antidepressants.

The strength of this trial is the probiotic strains and prebiotic types which have been previously found psychotropic in human $^{13,39,43}$ and animal studies. ${ }^{22,30}$ In addition we used BDI-II to measure depression severity which is well validated in psychiatric studies and in Iranian population. ${ }^{24}$ However, a number of limitations need to be considered. The major limitation of this study is not addressing the intestinal microbiota composition or sex hormones in the patients which possibly cause interactions. ${ }^{44,45}$ In addition, the patients were under prescription of different antidepressants. Although the medications belong to the same antidepressant group (SSRI), recruitment of patients who are taking the same medication could be preferable.

It is conceivable that the probiotic may ameliorate depression through a mechanism other than BDNF and the depression improvement can actually mediate the effect of the probiotic on BDNF, and to confirm the BDNF increase as an underlying mechanism of probiotics, further studies with measurement of these parameters in some points between start and end of the study is recommended. It will reveal whether the change in serum BDNF before the depression symptoms is improved. However this seems unlikely, since increase in BDNF levels is found to be independent of depression improvement in a previous study which showed that BDNF levels increased while total depression scores was not significantly different between the control group (conventional drug therapy) and treatment group (probiotic with electroacupuncture). ${ }^{46}$

\section{Conclusions}

This post hoc analysis revealed that supplementation with this 
psychobiotic formulation for 8 weeks in patients with low to moderate depression resulted in significantly higher serum BDNF levels, while prebiotic and placebo had no significant effect. In the same cohort, we had previously shown that this probiotic supplement containing L. helveticus R0052 and B. longum R0175 exerted a beneficial effect in improving depression scores compared to placebo, while the effects of prebiotic supplement were not major. The results of this study suggest that specific probiotics may improve MDD partially via BDNF. More clinical trials are needed to confirm the possible beneficial effect of other probiotics and prebiotics on BDNF levels and depression in psychiatric populations.

\section{Supplementary Materials}

Note: To access the supplementary tables and figure mentioned in this article, visit the online version of Journal of Neurogastroenterology and Motility at http://www.jnmjournal.org/, and at https:// doi.org/10.5056/jnm20079.

Financial support: This study was supported by the Vice Chancellor of Research, Tehran University of Medical Sciences (Grant No. 94-02-16134937); the experimental products were provided by Lallemand Health Solutions (Mirabel, QC, Canada).

\section{Conflicts of interest: None.}

Author contributions: Asma Kazemi and Kurosh Djafarian designed the research study; Nazanin Heidarzadeh-Rad contributed to the design; Nazanin Heidarzadeh-Rad, Asma Kazemi, and Kurosh Djafarian performed the research, analyzed, and interpreted the data; Nazanin Heidarzadeh-Rad drafted the manuscript; Kurosh Djafarian had primary responsibility for the final content; and all authors critically revised the manuscript for important intellectual content and approved the final manuscript.

\section{References}

1. Dinan TG, Stanton C, Cryan JF. Psychobiotics: a novel class of psychotropic. Biol Psychiatry 2013;74:720-726.

2. Bermúdez-Humarán LG, Salinas E, Ortiz GG, Ramirez-Jirano LJ, Morales JA, Bitzer-Quintero OK. From probiotics to psychobiotics: live beneficial bacteria which act on the brain-gut axis. Nutrients 2019;11:890.

3. Ibáñez CF. Neurotrophic factors: from structure-function studies to designing effective therapeutics. Trends Biotechnol 1995;13:217-227.

4. Pérez-Navarro E, Gavaldà N, Gratacòs E, Alberch J. Brain-derived neurotrophic factor prevents changes in Bcl-2 family members and caspase- 3 activation induced by excitotoxicity in the striatum. J Neurochem
2005;92:678-691.

5. Fossati P, Radtchenko A, Boyer P. Neuroplasticity: from MRI to depressive symptoms. Eur Neuropsychopharmacol 2004;14(suppl 5):S503S5 10 .

6. Lee JG, Park MK, Kim YH, et al. The correlations among depressive symptoms, cognitive performance and serum BDNF levels in the patients with chronic kidney disease. Psychiatry Investig 2018;15:186-192.

7. Numakawa T, Suzuki S, Kumamaru E, Adachi N, Richards M, Kunugi H. BDNF function and intracellular signaling in neurons. Histol Histopathol 2010;25:237-258.

8. Wang H, Lee IS, Braun C, Enck P. Effect of probiotics on central nervous system functions in animals and humans: a systematic review. $\mathrm{J}$ Neurogastroenterol Motil 2016;22:589-605.

9. Sudo N, Chida Y, Aiba Y, et al. Postnatal microbial colonization programs the hypothalamic-pituitary-adrenal system for stress response in mice. J Physiol 2004;558(Pt 1):263-275.

10. Cryan JF, Dinan TG. Mind-altering microorganisms: the impact of the gut microbiota on brain and behaviour. Nat Rev Neurosci 2012;13:701712 .

11. Keightley PC, Koloski NA, Talley NJ. Pathways in gut-brain communication: evidence for distinct gut-to-brain and brain-to-gut syndromes. Aust N Z J Psychiatry 2015;49:207-214.

12. Bercik P, Verdu EF, Foster JA, et al. Chronic gastrointestinal inflammation induces anxiety-like behavior and alters central nervous system biochemistry in mice. Gastroenterology 2010;139:2102-2112, e1.

13. Messaoudi M, Lalonde R, Violle N, et al. Assessment of psychotropiclike properties of a probiotic formulation (Lactobacillus helveticus R0052 and Bifidobacterium longum R0175) in rats and human subjects. Br J Nutr 2011;105:755-764.

14. Benton D, Williams C, Brown A. Impact of consuming a milk drink containing a probiotic on mood and cognition. Eur J Clin Nutr 2007;61:355-361.

15. Steenbergen L, Sellaro R, van Hemert S, Bosch JA, Colzato LS. A randomized controlled trial to test the effect of multispecies probiotics on cognitive reactivity to sad mood. Brain Behav Immun 2015;48:258-264.

16. McFarland LV, Evans CT, Goldstein EJ. Strain-specificity and diseasespecificity of probiotic efficacy: a systematic review and meta-analysis. Front Med (Lausanne) 2018;5:124.

17. Stenman LK, Patterson E, Meunier J, Roman FJ, Lehtinen MJ. Strain specific stress-modulating effects of candidate probiotics: a systematic screening in a mouse model of chronic restraint stress. Behav Brain Res 2020;379:112376.

18. Dinan TG, Cryan JF. Brain-gut-microbiota axis and mental health. Psychosom Med 2017;79:920-926.

19. Diop L, Guillou S, Durand H. Probiotic food supplement reduces stress-induced gastrointestinal symptoms in volunteers: a double-blind, placebo-controlled, randomized trial. Nutr Res 2008;28:1-5.

20. Arseneault-Bréard J, Rondeau I, Gilbert K, et al. Combination of Lactobacillus helveticus R0052 and Bifidobacterium longum R0175 reduces post-myocardial infarction depression symptoms and restores intestinal permeability in a rat model. Br J Nutr 2012;107:1793-1799.

21. Gilbert K, Arseneault-Bréard J, Flores Monaco F, et al. Attenuation of 
post-myocardial infarction depression in rats by $\mathrm{n}-3$ fatty acids or probiotics starting after the onset of reperfusion. Br J Nutr 2013;109:50-56.

22. Ait-Belgnaoui A, Colom A, Braniste V, et al. Probiotic gut effect prevents the chronic psychological stress-induced brain activity abnormality in mice. Neurogastroenterol Motil 2014;26:510-520.

23. Kazemi A, Noorbala AA, Azam K, Eskandari MH, Djafarian K. Effect of probiotic and prebiotic vs placebo on psychological outcomes in patients with major depressive disorder: a randomized clinical trial. Clin Nutr 2019;38:522-528.

24. Ghassemzadeh H, Mojtabai R, Karamghadiri N, Ebrahimkhani N. Psychometric properties of a persian-language version of the Beck Depression Inventory-Second edition: BDI-II-PERSIAN. Depress Anxiety 2005;21:185-192.

25. Taraz M, Khatami MR, Dashti-Khavidaki S, et al. Sertraline decreases serum level of interleukin-6 (IL-6) in hemodialysis patients with depression: results of a randomized double-blind, placebo-controlled clinical trial. Int Immunopharmacol 2013;17:917-923.

26. Kazemi A, Noorbala A, Djafarian K. Effect of probiotic and prebiotic versus placebo on appetite in patients with major depressive disorder: post hoc analysis of a randomised clinical trial. J Hum Nutr Diet 2020;33:5665.

27. Björkholm C, Monteggia LM. BDNF-a key transducer of antidepressant effects. Neuropharmacology 2016;102:72-79.

28. Wolkowitz OM, Wolf J, Shelly W, et al. Serum BDNF levels before treatment predict SSRI response in depression. Prog Neuropsychopharmacol Biol Psychiatry 2011;35:1623-1630.

29. Romijn AR, Rucklidge JJ, Kuijer RG, Frampton C. A double-blind, randomized, placebo-controlled trial of Lactobacillus helveticus and Bifidobacterium longum for the symptoms of depression. Aust N Z J Psychiatry 2017;51:810-821.

30. Liang S, Wang T, Hu X, et al. Administration of Lactobacillus helveticus NS8 improves behavioral, cognitive, and biochemical aberrations caused by chronic restraint stress. Neuroscience 2015;310:561-577.

31. Bercik P, Park AJ, Sinclair D, et al. The anxiolytic effect of Bifidobacterium longum NCC3001 involves vagal pathways for gut-brain communication. Neurogastroenterol Motil 2011;23:1132-1139.

32. Klein AB, Williamson R, Santini MA, et al. Blood BDNF concentrations reflect brain-tissue BDNF levels across species. Int J Neuropsychopharmacol 2011;14:347-353.

33. Pan W, Banks WA, Fasold MB, Bluth J, Kastin AJ. Transport of brainderived neurotrophic factor across the blood-brain barrier. Neuropharmacology 1998;37:1553-1561.

34. Pinto-Sanchez MI, Hall GB, Ghajar K, et al. Probiotic Bifidobacterium longum NCC3001 reduces depression scores and alters brain activity: a pilot study in patients with irritable bowel syndrome. Gastroenterology 2017;153:448-459, e8.

35. Haghighat N, Rajabi S, Mohammadshahi M. Effect of synbiotic and probiotic supplementation on serum brain-derived neurotrophic factor level, depression and anxiety symptoms in hemodialysis patients: a randomized, double-blinded, clinical trial. Nutr Neurosci 2019:1-10.

36. Vaziri ND, Zhao YY, Pahl MV. Altered intestinal microbial flora and impaired epithelial barrier structure and function in CKD: the nature, mechanisms, consequences and potential treatment. Nephrol Dial Transplant 2015;31:737-746.

37. Chung YC, Jin HM, Cui Y, et al. Fermented milk of Lactobacillus helveticus IDCC3801 improves cognitive functioning during cognitive fatigue tests in healthy older adults. J Funct Foods 2014;10:465-474.

38. Akkasheh G, Kashani-Poor Z, Tajabadi-Ebrahimi M, et al. Clinical and metabolic response to probiotic administration in patients with major depressive disorder: a randomized, double-blind, placebo-controlled trial. Nutrition 2016;32:315-320.

39. Messaoudi M, Violle N, Bisson JF, Desor D, Javelot H, Rougeot C. Beneficial psychological effects of a probiotic formulation (Lactobacillus helveticus R0052 and Bifidobacterium longum R0175) in healthy human volunteers. Gut microbes 2011;2:256-261.

40. Davis LM, Martínez I, Walter J, Hutkins R. A dose dependent impact of prebiotic galactooligosaccharides on the intestinal microbiota of healthy adults. Int J Food Microbiol 2010;144:285-292.

41. Bastiaanssen TF, Cussotto S, Claesson MJ, Clarke G, Dinan GT, Cryan JF. Gutted! unraveling the role of the microbiome in major depressive disorder. Harv Rev Psychiatry 2020;28:26-39.

42. Munoz-Bellido JL, Munoz-Criado S, Garcì-Rodrìguez JA. Antimicrobial activity of psychotropic drugs: selective serotonin reuptake inhibitors. Int J Antimicrob Agents 2000;14:177-180.

43. Savignac HM, Corona G, Mills H, et al. Prebiotic feeding elevates central brain derived neurotrophic factor, N-methyl-d-aspartate receptor subunits and D-serine. Neurochem Int 2013;63:756-764.

44. Audet MC. Stress-induced disturbances along the gut microbiotaimmune-brain axis and implications for mental health: does sex matter? Front Neuroendocrinol 2019;54:100772.

45. Tsilimigras MC, Gharaibeh RZ, Sioda M, Gray L, Fordor AA, Lyte M. Interactions between stress and sex in microbial responses within the microbiota-gut-brain axis in a mouse model. Psychosom Med 2018;80:361369.

46. Huang W, Gan W, Huang A, et al. Efficacy of electroacupuncture combined with probiotics for depression and chronic diarrhea in patients and effect on serum inflammatory cytokines, NE and BDNF. Exp Ther Med 2019;18:3470-3474. 